Original Research Paper

\title{
Optimized Energy Efficient Virtual Machine Placement Algorithm and Techniques for Cloud Data Centers
}

\author{
${ }^{1}$ Sanjay Patel and ${ }^{2}$ Ramji M. Makwana \\ ${ }^{I}$ Department of Computer Engineering, LDRP-ITR, CHARUSAT, Gandhinagar, Changa, India \\ ${ }^{2}$ Department of Information Technology, V.V.P. College of Engineering, Rajkot, India
}

\author{
Article history \\ Received: 09-06-2016 \\ Revised: 07-11-2016 \\ Accepted: 24-11-2016 \\ Corresponding Author: \\ Sanjay Patel \\ Department of Computer \\ Engineering, LDRP-ITR, \\ CHARUSAT, Gandhinagar, \\ Changa, India \\ Email: sanjaypatel54@gmail.com
}

\section{Introduction}

Cloud technology aims to provide pay per use service, reliable, Specified resources and QoS services for the consumers and end users. There are several companies provide cloud computing services to the end users (Feller et al., 2011).

Using several fundamental models cloud providers offers various services for the users according to their requirements:

- Software as a service (SaaS)

- Platform as a service (PaaS)

- Infrastructure as a service (IaaS)

There are four deployments in cloud in Fig. 1 (Mell and Grance, 2014):

- Public cloud

- Private cloud

- Community cloud

- Hybrid cloud

\begin{abstract}
Cloud computing is an internet based computing technology that provide on demand computing for end users. Normally, data centers allocation for application on statically based. But today so many data centers have a problem how to reduce energy consumption? Due to increase use of cloud services and infrastructure by various cloud providers, uses of energy day by day increase that's why energy consumption increase lots. Large numbers of data centers that consume lots of energy which increase the level of co2. Hence there is need for green computing and for energy efficient management of cloud data center resources besides meeting the QoS constraints. Within a data center, maximum energy is consumed by cooling systems and ICT Infrastructure, particularly, the servers. So it is of utmost importance to optimize energy utilization in datac enter servers. In order to achieve this, we can leverage the power of virtualization which is intrinsic in Cloud Computing. Virtualization opens the doors for VM consolidation by allowing dynamic migration of virtual machines across physical machines. In this study, the problem of VM consolidation has been formulated as a mathematical optimization problem.
\end{abstract}

Keywords: Cloud Computing, Virtualization, Allocation of Virtual Machines, Quality of Service (QoS), Energy Aware VM Allocation, Social Networking

\section{Why Energy-Aware Cloud Computing for Social Networking?}

"As per McKinsey report data centers approximate energy bill in 2010 is $\$ 11.5$ billion. Also this costs double every five years" (Buyya et al., 2010).

- Energy Efficient: Energy efficient in virtualized data centers provides QoS and also reduces the operational cost for the companies

- Energy Saving: As per current utilization of resources, topologies of network and thermal computing nodes achieved energy saving using VM migration and VM consolidation algorithms (Buyya et al., 2010)

The demand for Cloud infrastructure has expanded with rapid growth of computing applications and data. Hence more and more servers and disks are required to process them fast enough within the given time limit. Data centers are expensive to maintain and hostile to the environment. Over $90 \%$ of a data center's electricity is consumed by the IT equipment (server, storage and network) and cooling equipment (Minas and Ellison, 2009). 


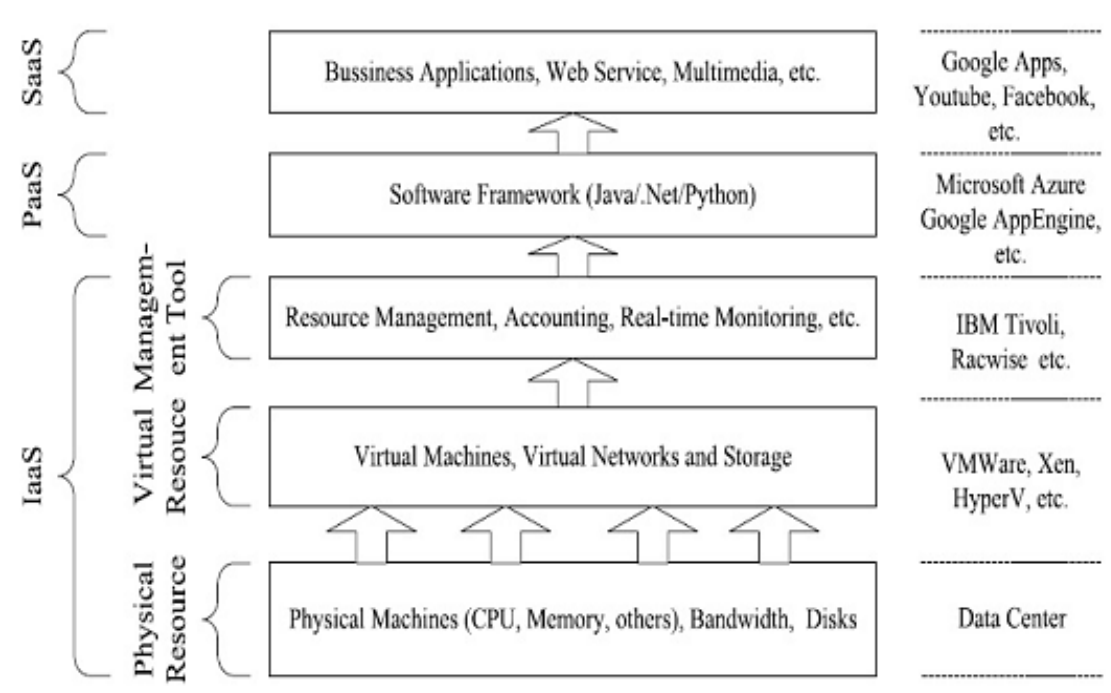

Fig. 1. Cloud computing architecture (Jing et al., 2013)

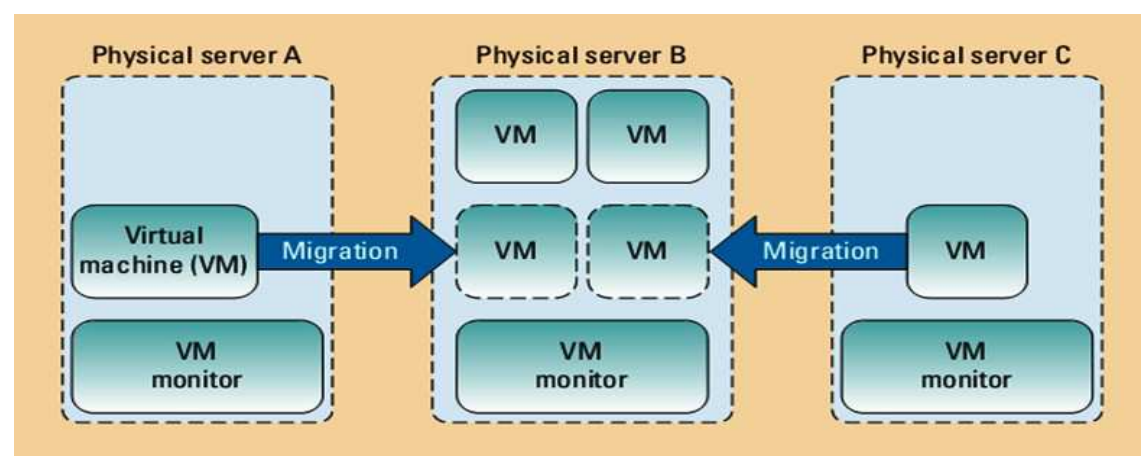

Fig. 2. VM Consolidation (Jing et al., 2013)

Substantial amount of electricity is needed to power and cool various servers in the data centers (Mell and Grance, 2014). The cloud providers have to make sure that profit margin is not intensely reduced due to high energy costs. The need for energy-efficient cloud computing arises due to (Qumranet Inc, 2014): Increased cost of ownership, Reduced Return on Investment, Increase in carbon footprint, Decreased reliability and availability of data center resources and, Pressure from the Govt. to reduce carbon footprint. This paves the way for Green Computing. Green computing, green IT or ICT Sustainability is the study and practice of environmentally sustainable computing or IT (Mell and Grance, 2014). San Murugesan notes that this can include "designing, manufacturing, using and disposing of computers, servers and associated subsystems-such as monitors, printers, storage devices and networking and communications systems efficiently and effectively with minimal or no impact on the environment" (Mell and Grance, 2014).

A number of challenges are being faced with respect to implementing energy efficient cloud computing.
These are (Mell and Grance, 2014): Energy aware dynamic resource allocation, QoS- based Resource Selection and Provisioning, Optimization of Virtual Network Topologies, Autonomic Optimization of Thermal states and Cooling System Operation, Efficient Consolidation of VMs for Managing Heterogeneous Work- loads. Most of these problems can be addressed with the concept of VM consolidation which in Fig. 2. As per (Wei et al., 2009) VM consolidation can be defined as the process of gathering several Virtual Machines (VMs) into a single physical server. This is made possible due to the concept of virtualization which introduces an abstraction layer between an OS and hardware. Physical resources can be divided into a number of logical pieces called Virtual Machines (VMs). Each VM can house an individual OS there by giving the user a view of a dedicated physical resource and guaranteeing the performance and failure isolation among VMs sharing a single physical machine. This allows reducing the amount of hardware in use and improving the utilization of resources. Now energy consumption can be reduced by 
turning off the unused hardware. VM consolidation can be either static or dynamic.

In this study, the problem of energy-efficient server utilization has been formulated as a mathematical optimization problem.

\section{Related Work}

The main aim of this work is to implement and development of energy-aware resource allocation techniques and defines various policies for data centers. So, that data centers become more eco-friendly to drive scientific, commercial and technological advancement for future generations. There are basically four main entities involved (Buyya et al., 2010):

- Consumers

- Green resource allocator and providers

- Virtual machines

- Physical machines or node

\section{Sources of Power Consumption}

As per Intel Research Labs report (Minas and Ellison, 2009) physical server is main part of power consumed which includes various Processors, RAM and losses due to the power supply inefficiency in Fig. 3. As per data show in figure the Processors no longer dominates power consumption by a physical server. That's why techniques required continuous improvement of the processor power efficiency and application of power saving techniques that enable active low-power modes.

As per some report multi-core processors are much more efficient than conventional architectures. There are another reason to reduce the power consumed by $\mathrm{CPU}$ relatively to the whole system is adoption of multi-core architectures.

The problem of VM consolidation can be subdivided into four sub-problems (Buyya et al., 2010). These are shown in the Fig. 3. We will limit our discussion to VM placement strategies. Wei et al. (2009) have presented Dynamic consolidation with migration control.

We will limit our discussion to VM placement strategies. Wei et al. (2009) have presented Dynamic consolidation with migration control in Fig. 4.

Their aim is to ensure more stability for VMs with steady demands and that VMs with steady demand do not migrate. They have contributed an LP formulation and heuristics to control VM migration, which prioritize VMs with steady capacity.

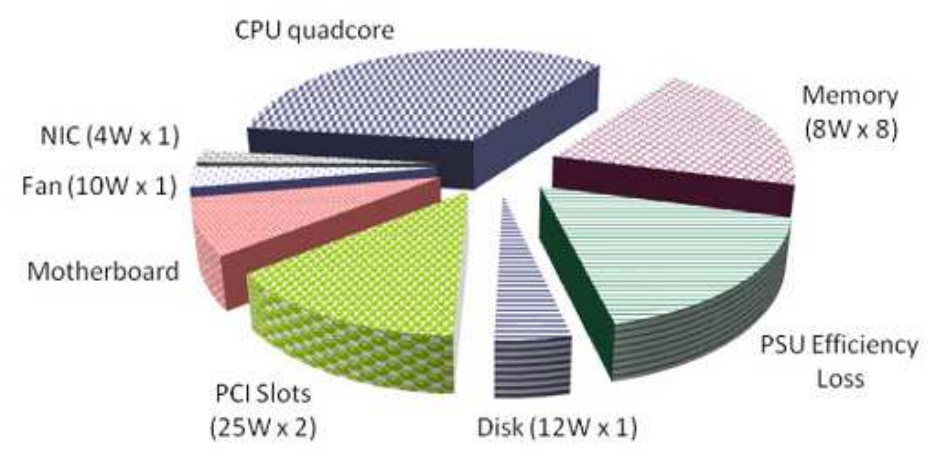

Fig. 3. Sources of power consumption (Beloglazov et al., 2010)

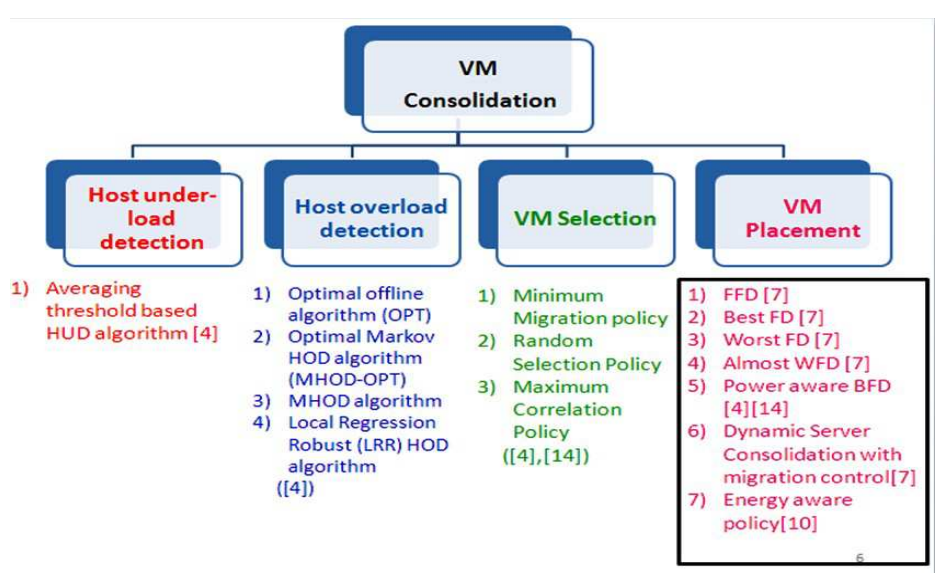

Fig. 4. Subdivision of VM consolidation problem 
Buyya et al., (2010; Beloglazov et al., 2010) has discussed the Power-aware BFD algorithm for VM placement which is a modification of the BFD algorithm. In this algorithm each VM is allocated to a host for which the increase in power consumption caused by the allocation is the smallest. Wu et al. (2011) have presented Energy aware management policy. They have proposed the usage of energy assessments to optimize VM placement for achieving energy efficiency. They have also formulated an optimization problem of managing IaaS providers in private Cloud scenarios while considering energy efficiency. Beloglazov et al. (2010) have formulated a multi- objective optimization problem for VM placement problem. Ghribi et al. (2013) have discussed capacity planning problems in the context of virtualized IT infrastructures and decision models for optimal allocation of virtual servers. The existing VM Placement policies discussed have the following disadvantages:

- The algorithms don't take into account the type of workloads at destination data center node. Combining different types of workloads can help improve the CPU utilization of a node

- Distance between the candidate node for migration and destination node can be considered as an important factor for choosing destination node, to consider communication cost. This too has not been considered in the algorithms studied so far

\section{Energy Consumption in Computing Systems}

In world there are large number of researchers which are works for to develop techniques and methods for decrease of the energy consumption in physical data centres in Fig. 5 (Beloglazov et al., 2010) [12].

\section{Virtualized Technology Vendors}

There are three types of virtualization technology solutions.

Xen

Xen hypervisor is one of the open source virtualization technology developed by the Xen community (Wei et al., 2009).

\section{VMware}

VMware offered VMware ESX Server and VMware ESXi enterprise level virtualization solutions (VMware Inc, 2015).

\section{$K V M$}

As a module or part of Linux kernel (Open Source) KVM provides virtualization platform (Qumranet Inc, 2014).

\section{Virtualized Systems}

- VirtualPower: Coordinated power management

- Coordinated multi-level power management

- Resource allocation using virtual clusters

- pMapper: Migration and power cost aware application placement

- GreenCloud: SLA and energy-efficient based cloud resources management

\section{Using the DVFS Green Energy-Efficient Scheduling Algorithm}

The system architecture shown in Fig. 6 consists of the Submit Job, the VMM, Scheduling Algorithm, Various Servers and Virtual Machines and the DVFS controller for power saving [12].

The following steps are there (Wu et al., 2011):

- Job submission: To submit job from users

- VM manager: To manage VMs as well as application

- Scheduling Algorithm: To schedule job

- DVFS controller: For power saving

- Servers and VMs: Includes physical machines and virtual machines

The main objective of this work is using green energy-efficient scheduling algorithm perform job scheduling for Cloud computing. To select various VMs for executing jobs is priority for job scheduling algorithms in Fig. 6 (Wu et al., 2011).

\section{Energy-Aware Model}

There are main three parts are there for energy-aware models (Ghribi et al., 2013):

- Energy-aware VM scheduler

- Energy consumption estimator

- Cloud iaas manager

\section{Algorithms}

There are main three types of algorithms are there in Fig. 7 (Ghribi et al., 2013):

- Exact VM Allocation Algorithm

- Exact VM Migration Algorithm

- Adapted Energy-aware Best Fit Algorithm

\section{Problem Formulation}

The problem of energy-efficient server utilization in cloud data centers can be formulated as an optimization problem. The objective would be to minimize the total number of powered-on servers in data center. 


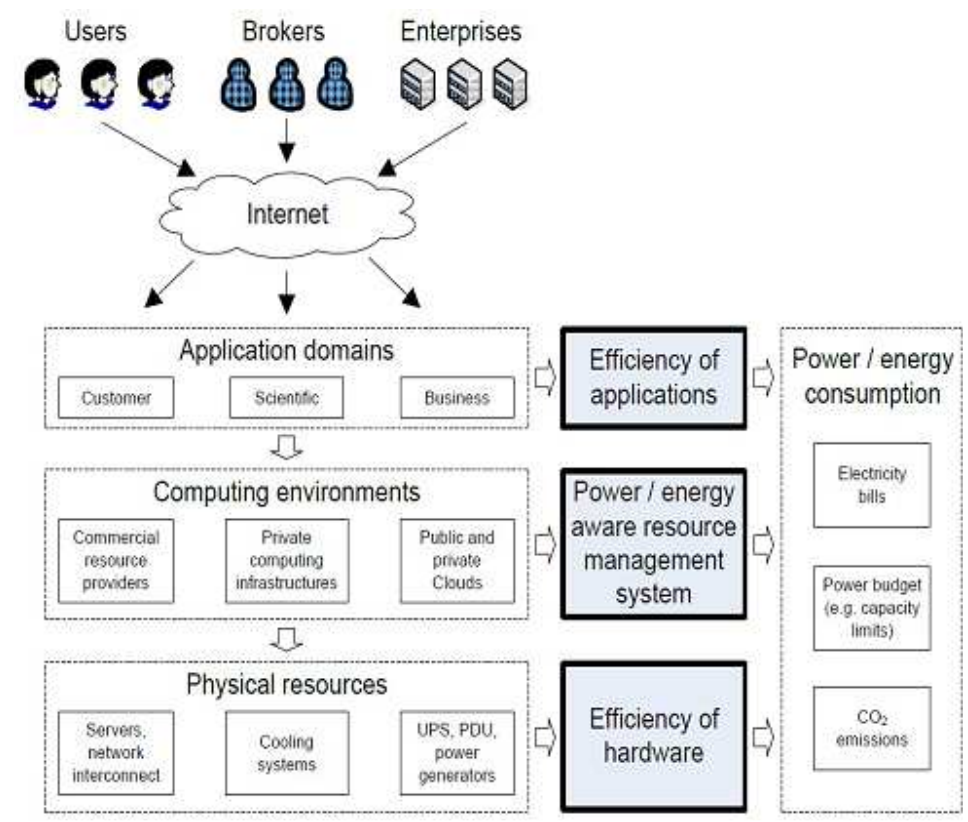

Fig. 5. Energy consumption in computing systems (Beloglazov et al., 2010)

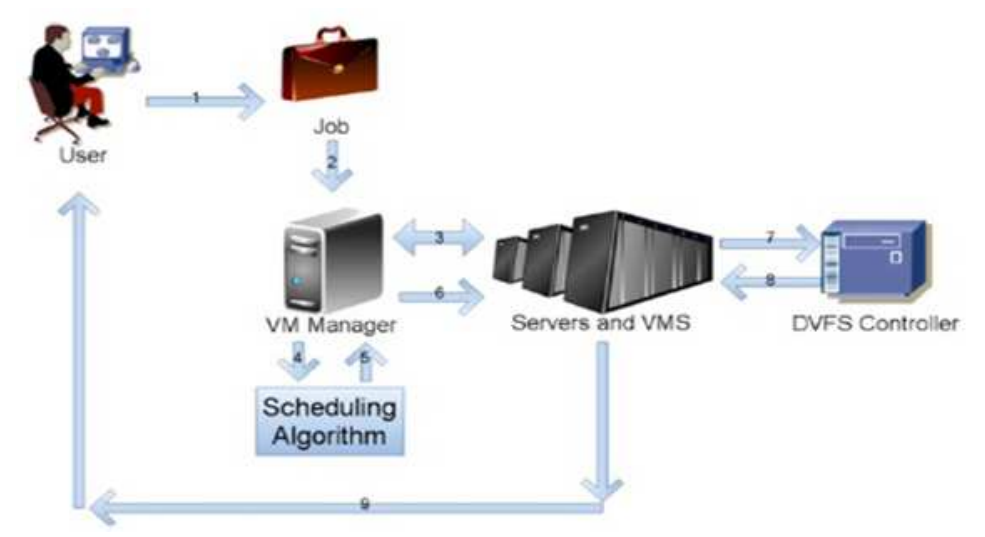

Fig. 6. The system architecture using DVFS controller (Wu et al., 2011)

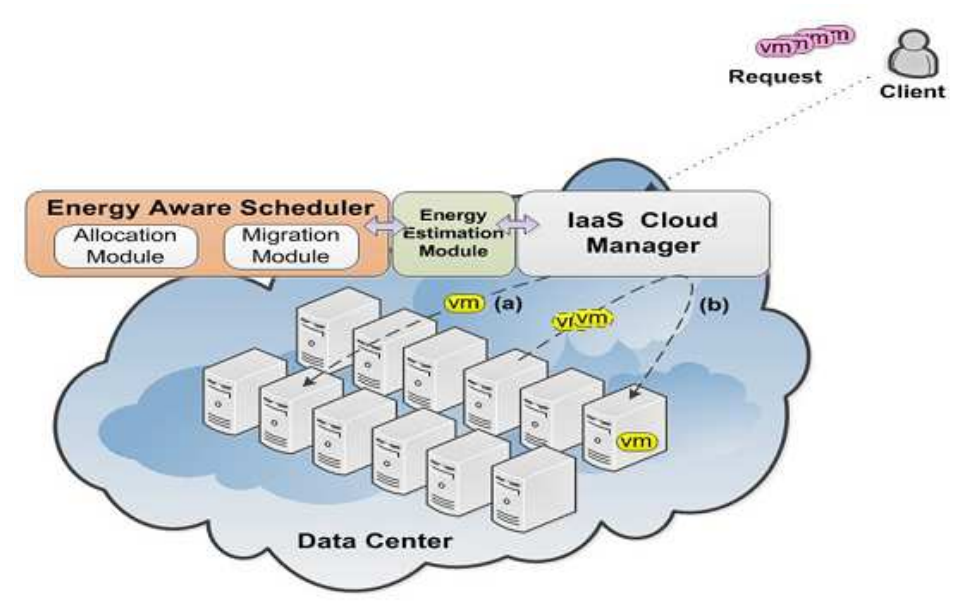

Fig. 7. Energy-aware model (Ghribi et al., 2013) 
Table 1. Parameters

\begin{tabular}{ll}
\hline Parameter & Description \\
\hline$n$ & \#VMs \\
$m$ & \#Physical machines (PMs) \\
$I$ & Set of all VMs \\
$J$ & Set of all PMs \\
$R^{P}{ }_{i t}$ & CPU demand of VM I at time $t$ \\
$T^{L}{ }_{j}$ & Lower bound on CPU utilization (to eliminate \\
& the idle power consumption) \\
$T^{U}{ }_{j}$ & upper bound on CPU utilization (to prevent \\
& SLA violation) \\
$x_{i j t}$ & A binary variable which indicates if VM $i$ is \\
& assigned to server $j$ at time $t$ \\
$y_{j}$ & the binary variable which indicates whether \\
& server j is in use or not. \\
$t$ & Time is divided into discrete time steps \\
& indicated by $t=\{1, \ldots, \tau\}$ \\
$M$ & Maximum VM Migration Count (VMMC) \\
\hline
\end{tabular}

The constraints will ensure that at any time, the utilization of a server is within the predefined lower- and upper-threshold. This will prevent under-utilization and over-utilization of the data center servers.

This in turn ensures that the server resources are not wasted or overburdened (which will affect the longevity) and that energy consumption is minimal. The constraints will also put a limit on the number of times a particular VM can be migrated during a specified time interval. This will prevent thrashing. The formulation and the parameters in the Table 1:

$$
\text { Minimize } \sum_{j=1}^{m} y_{i}
$$

Subject to:

$$
\begin{aligned}
& T_{j}^{L} y i \leq \sum_{i=1}^{n} x_{i j t} \times R_{i t}^{P} \leq T_{j}^{U} y i, \forall j \in J \\
& x_{i j t}=1, \forall t \in \tau \\
& \sum_{t=2}^{\tau}\left|x_{i j t}-x_{i j t-1}\right| \leq M, \forall i \in I, \forall j \in J \\
& x_{i j t} \in\{0,1\}, \forall i \in I, \forall j \in J, \forall t \in \tau \\
& y_{j} \in\{0,1\}, \forall j \in J
\end{aligned}
$$

As per Equation 1, our objective is to minimize the total number of servers presently running in the data center, subject to a number of constraints. First constraint Equation 2 is that, the total CPU utilization at a server should be within the pre-specified upper bound $\left(T_{j}^{U}\right)$ and lower bound $\left(T^{L}{ }_{j}\right)$. If not the server is either under loaded $\left(T_{j y}^{L}>P n_{i}=1 x_{i j t} \mathrm{R}^{\mathrm{P}}\right.$ it $)$ or overloaded $P n_{i}=$ $\left.1 x_{i j t} R_{i t}^{P}>T_{j y j}^{U}\right)$. The second constraint, Equation 3 says that at a particular time a particular VM can belong to exactly one server. The third constraint, Equation 4 puts a limit on the number of migrations of a particular VM. This is done by making sure that the total number of migrations of a VM in time duration does not exceed M. The expression $x_{i j t} x_{i j t-1}$ gives the count of the number of migrations of VM $V M_{i j}$ in time duration. Constraint Equation 5 and 6 define the scope of variable $x_{i j t}$ and $y_{j}$.

\section{Conclusion}

Cloud computing technology is expected to grow and provide large services and computational power to end users. In this context energy efficiency is more important for virtualized data centers. Due to more energy consumption operating cost higher and co2 emission to the environment. From this paper identify all existing algorithms for energy aware and efficiency. Also find out research objective and scope in the field of energyaware virtualized data centers. In particular Literature survey presented in details all existing paper review, analysis of methods and comparative study between existing algorithms and techniques. Using literature survey to identify research gaps, open challenges and clearly determine the research direction. It can be concluded that energy efficient management of cloud data centers is indeed important and hence suitable techniques are required for energy efficient VM allocation. Virtualization is a very powerful technology which enables to address this concern using VM consolidation. In future we plan to propose an algorithm for $\mathrm{VM}$ consolidation to as per the formulation presented here.

\section{Acknowledgement}

The authors would like to thank the management of LDRP Institute of Technology and Research, Gandhinagar, India and the management of CSPIT, Charotar University of Science and Technology, Changa, India for providing necessary requirements in carrying out the research work. Special thank goes to Dr. Amit Ganatra, Dean, Charusat, Dr. Rajkumar Buyya, Director, CLOUDS Laboratory, Dr. Anton Beloglazov, Researcher, Google, Dr. S. Rama Mohan, IITRAM and Dr. D.G. Thakore, BVM, India for their able guidance and motivation. We are also thankful to Ms. Nimisha Thacker, LDRP Institute of Technology and Research, Gandhinagar, India for providing valuable inputs during this research work. We would also like to thank the anonymous reviewers and the editors.

\section{Author's Contributions}

Sanjay Patel: All experiments, Data Analysis, Algorithms. 
Ramji M. Makwana: Design research plan. Data Work, Guidance and Motivation.

\section{Ethics}

This article is original and contains unpublished material. The corresponding author confirms that all of the other authors have read and approved the manuscript and no ethical issues involved.

\section{References}

Beloglazov, A., R. Buyya, Y.C. Lee and A. Zomaya, 2010. A taxonomy and survey of energy-efficient data centers and cloud computing systems. The University of Melbourne.

Buyya, R., A. Beloglazov and J. Abawajy, 2010. Energy-efficient management of data center resources for cloud computing: A vision, architectural elements and open challenges. Proceedings of the International Conference on Parallel and Distributed Processiong Techniques and Applications, Jul. 12-15, Las Vegas, USA, pp: 1-12.

Feller, E., L. Rilling and C. Morin, 2011. Energy-aware ant colony based workload placement in clouds. Proceedings of the 12th IEEE/ACM International Conference on Grid Computing, Sept. 21-23, IEEE Xplore Press, pp: 26-33. DOI: 10.1109/Grid.2011.13
Ghribi, C., M. Hadji and D. Zeghlache, 2013. Energy efficient VM scheduling for cloud data centers: Exact allocation and migration algorithms. Proceedings of the 13th IEEE/ACM International Symposium on Cluster, Cloud and Grid Computing, May 13-16, IEEE Xplore Press, The Netherlands, pp: 671-678. DOI: 10.1109/CCGrid.2013.89

Jing, S.Y., S. Ali, K. She and Y. Zhong, 2013. Stateof-the-art research study for green cloud computing. J. Supercomput., 65: 445-468. DOI: $10.1007 / \mathrm{s} 11227-011-0722-1$

Mell, P. and T. Grance, 2014. The NIST defination of cloud computing.

Minas, L. and B. Ellison, 2009. Energy Efficiency for Information Technology: How to Reduce Power Consumption in Servers and Data Centers. 1st Edn., Intel Press, ISBN-10: 1934053201, pp: 330.

Qumranet Inc, 2014. KVM: Kernel-based virtualization driver. Qumranet Inc.

VMware Inc, 2015. vSphere resource management guide. VMware Inc.

Wei, G., J. Liu, J. Xu, G. Lu and K. Yu et al., 2009. The on-going evolutions of power management in Xen. Int. Corporat.

Wu, C.M., R.S. Chang and H.Y. Chan, 2011. A green energy-efficient scheduling algorithm using the DVFS technique for cloud data centers. Future Generat. Comput. Syst., 37: 141-147. DOI: 10.1016/j.future.2013.06.009 